\title{
Design Principles of NiFe Layered Double Hydroxide Anode Catalysts for Anion Exchange Membrane Water Electrolyzer
}

Sun Seo Jeon, Jinkyu Lim, Phil Woong Kang, Jae Won Lee, Gihun Kang, Hyunjoo Lee*

Department of Chemical and Biomolecular Engineering, Korea Advanced Institute of

Science and Technology (KAIST), Daejeon 34141, Republic of Korea

*E-mail: azhyun@kaist.ac.kr (H. L.)

Additional Data;

Figure S1 S16

Table S1 S3 


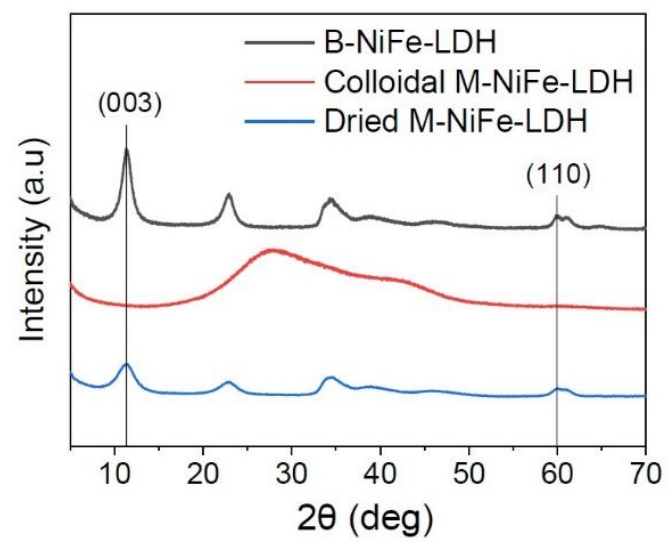

Figure S1. XRD patterns of B-NiFe-LDH, M-NiFe-LDH suspended in deionized water, M-

$\mathrm{NiFe}-\mathrm{LDH}$ dried in oven. 


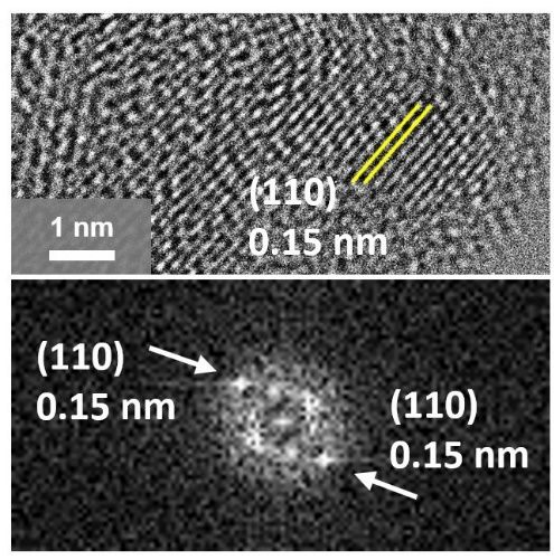

Figure S2. HR-TEM image (above) and FFT pattern (below) of M-NiFe-LDH. 
Table S1. The atomic composition of Ni and Fe in the B-NiFe-LDH and M-NiFe-LDH measured by ICP-MS.

\begin{tabular}{ccc}
\hline Catalyst & $\mathrm{Ni}$ & Fe [mole \%] \\
\hline B-NiFe-LDH & 76.24 & 23.76 \\
M-NiFe-LDH & 76.15 & 23.85 \\
\hline
\end{tabular}



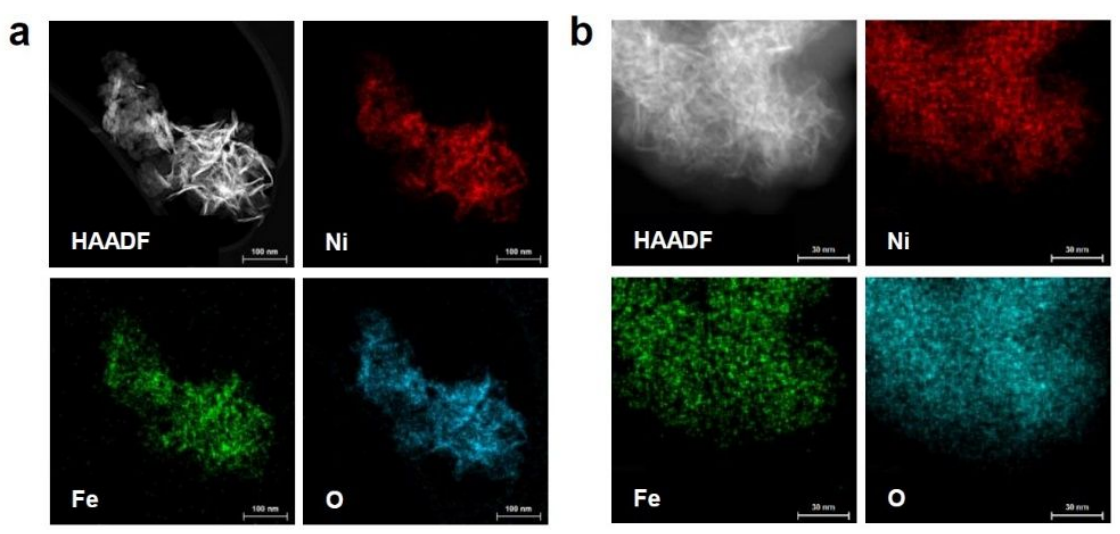

Figure S3. EDS mapping images of (a) B-NiFe-LDH and (b) M-NiFe-LDH. 

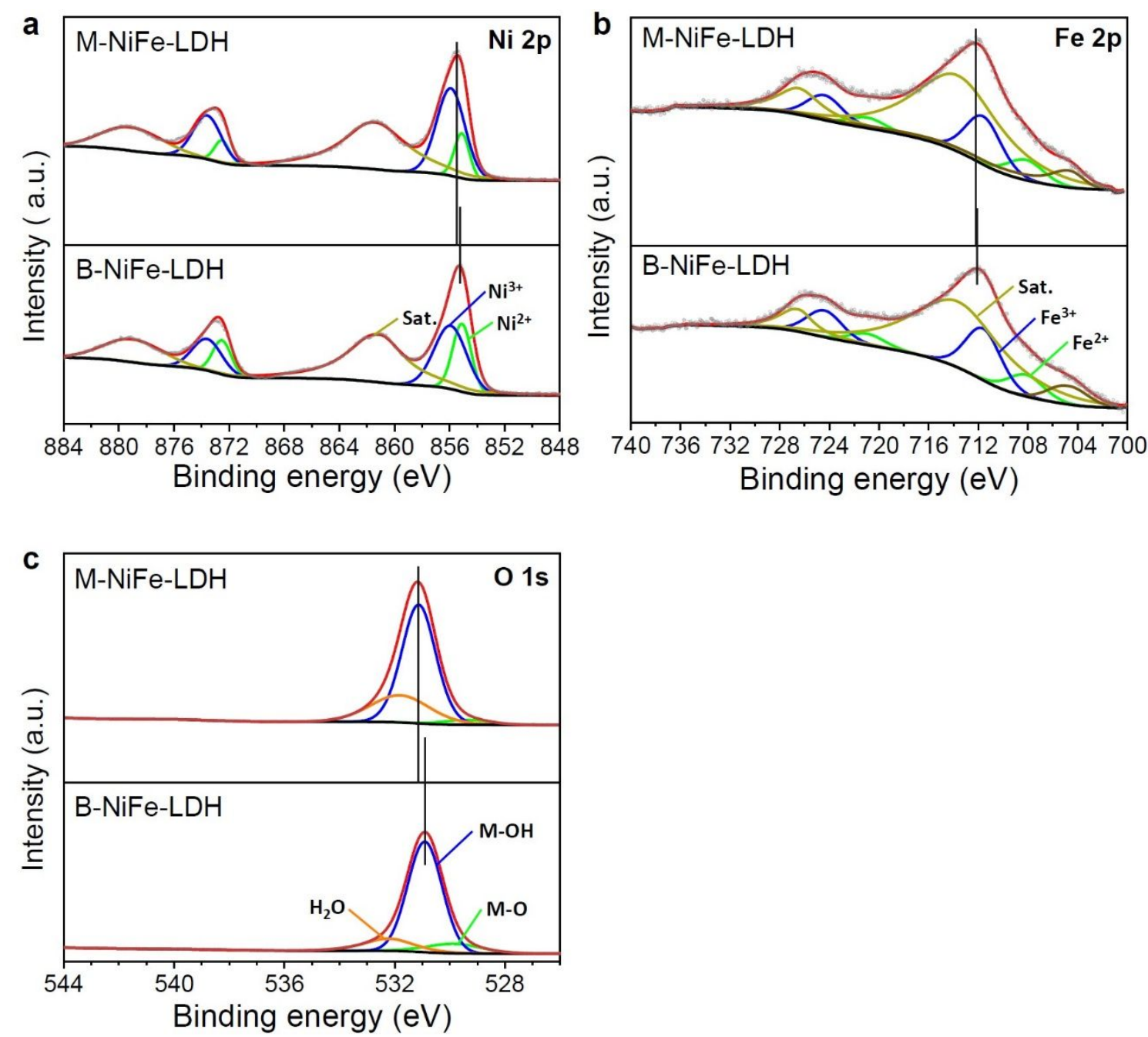

Figure S4. XPS spectra of (a) Ni 2p, (b) Fe 2p, and (c) O 1s for B-NiFe-LDH and M-NiFe-

LDH. 


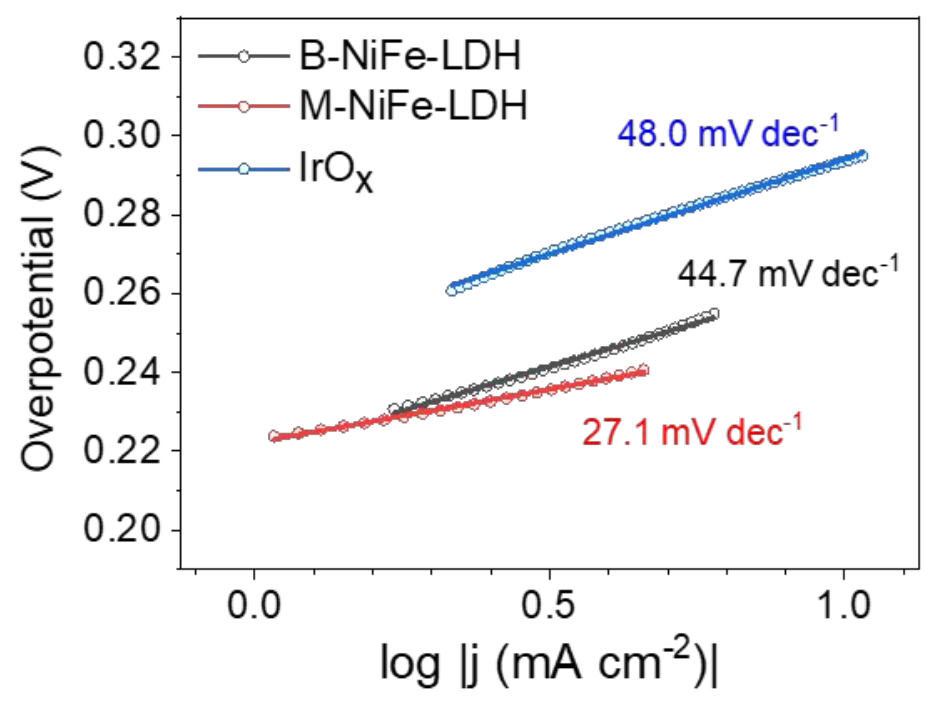

Figure S5. Tafel slopes obtained from the cathodic scan of cyclic voltammetry. 

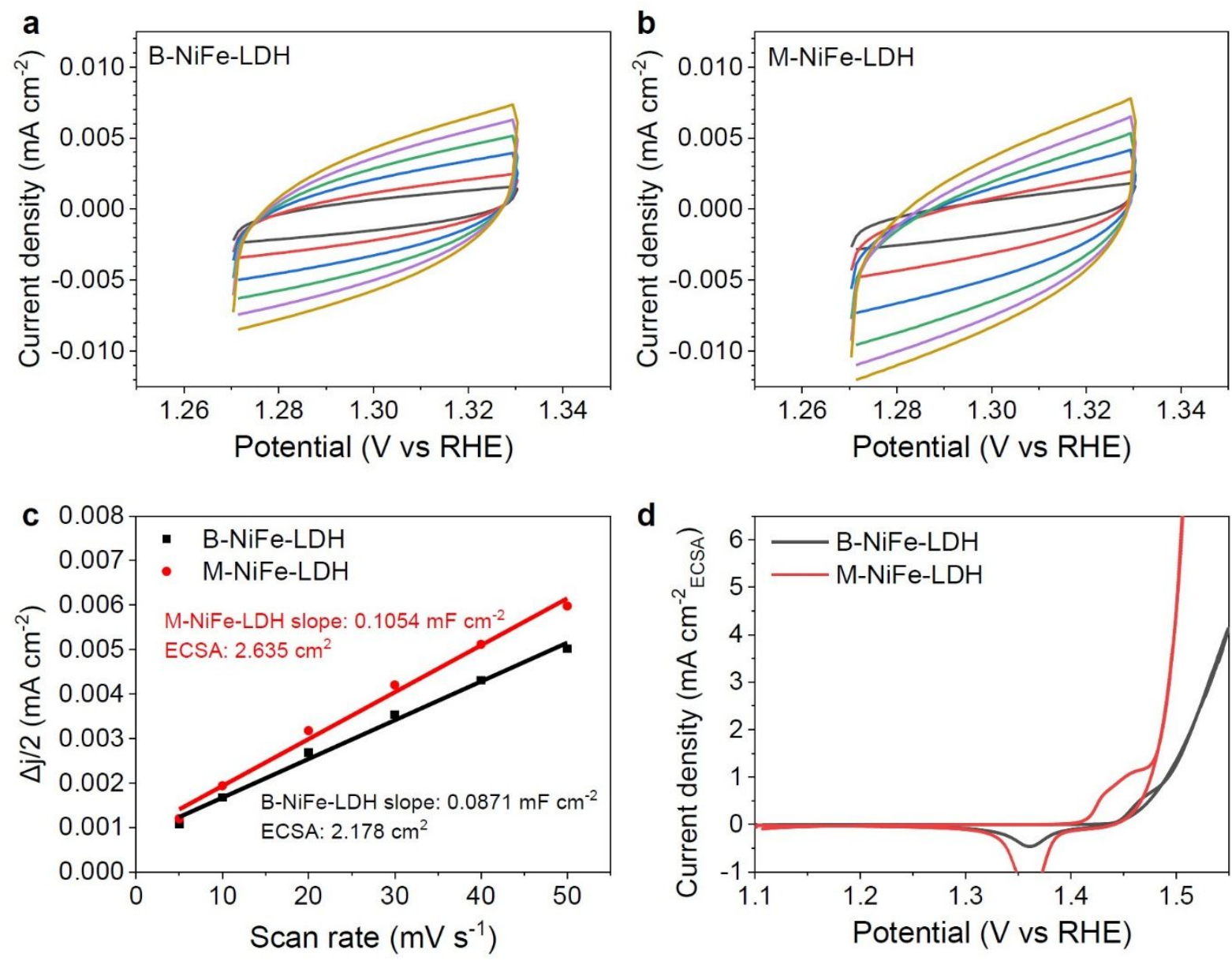

Figure S6. Estimating double layer capacitance (CDL) of (a) B-NiFe-LDH and (b) M-NiFe-

LDH by measuring CV curves in the non-faradaic current region at different scan rates (5;

black, 10; red, 20; blue, 30; green, 40; purple, 50; yellow $\mathrm{mV} \mathrm{s}^{-1}$ ), (c) the slopes of fitted lines in the 'current versus scan rate' relations, providing ECSA for the equal Fe content of 0.1068 mol, which was measured by ICP-MS, and (d) specific activity normalized by the ECSAs for B-NiFe-LDH and M-NiFe-LDH. 

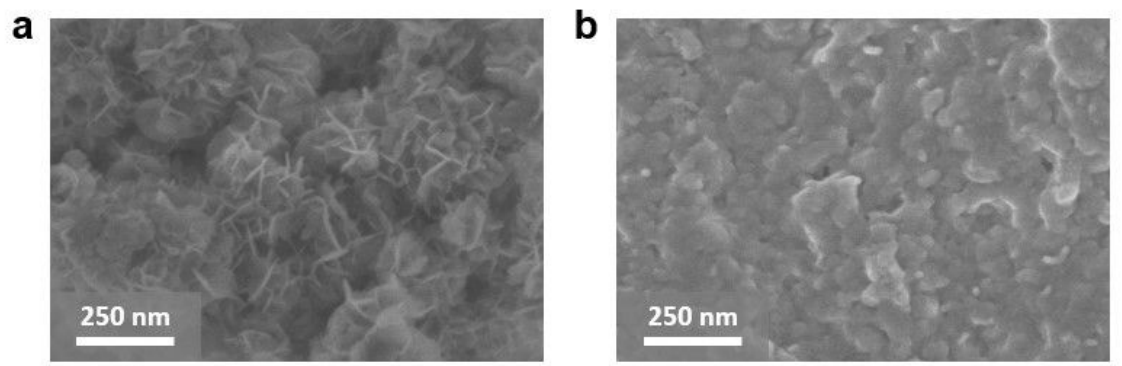

Figure S7. SEM images of (a) B-NiFe-LDH and (b) M-NiFe-LDH spray-coated on Ni foam at high magnification 

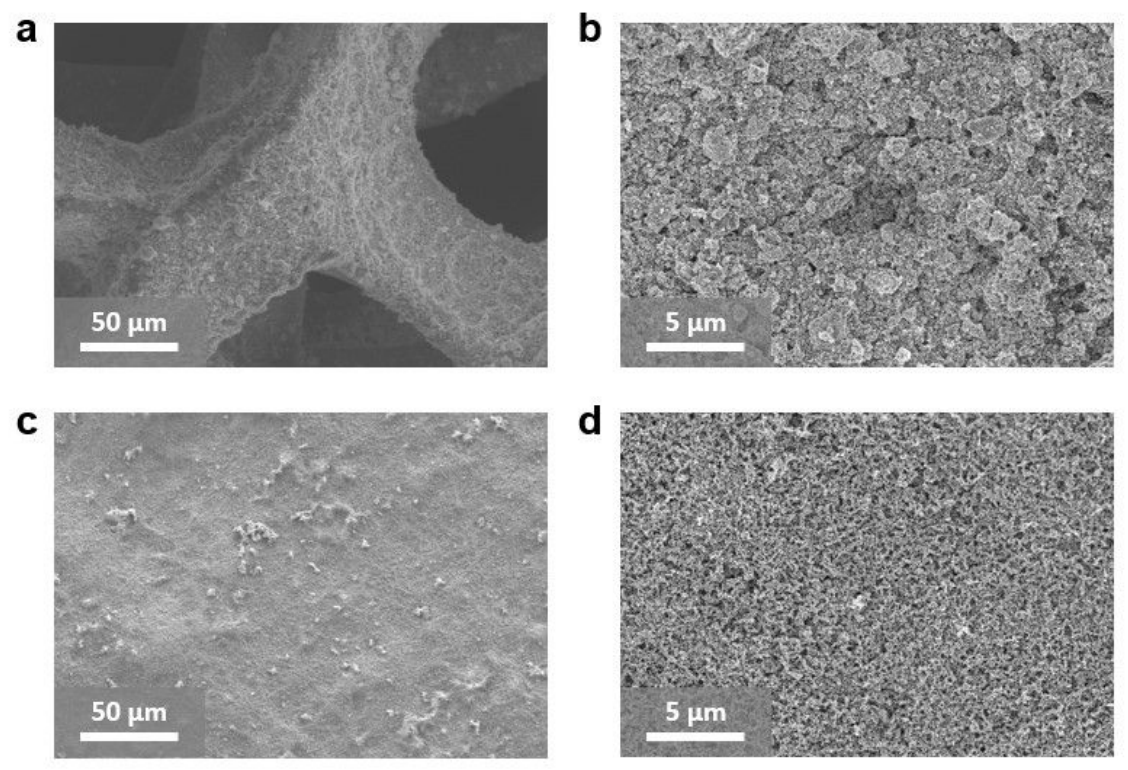

Figure S8. SEM images of (a), (b) $\mathrm{IrO}_{\mathrm{x}}$ sprayed on Ni foam, (c), (d) Pt/C sprayed on carbon paper. 


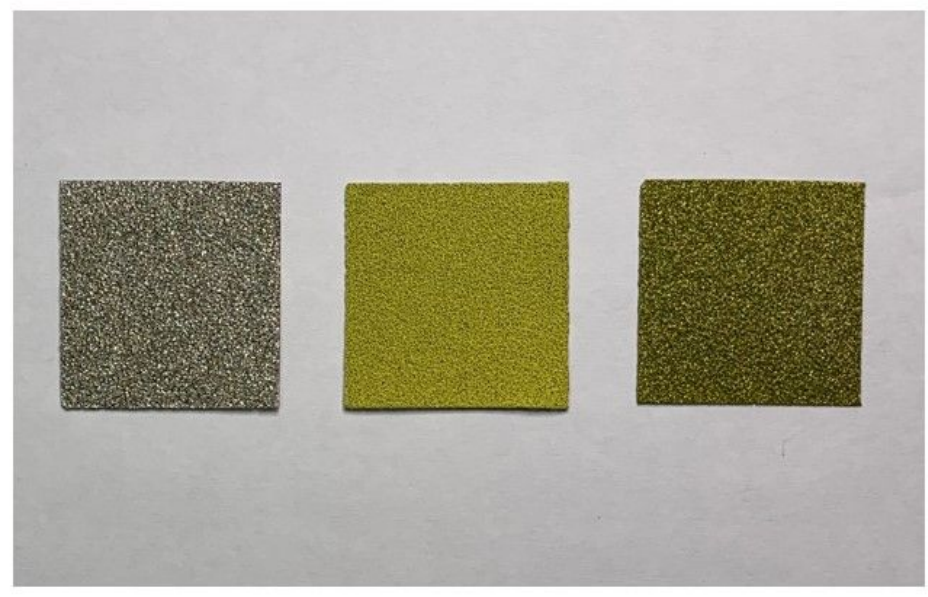

Figure S9. Photograph of (left) bare Ni foam, (middle) B-NiFe-LDH coated on Ni foam, and (right) $\mathrm{M}-\mathrm{NiFe}-\mathrm{LDH}$ coated on Ni foam. 

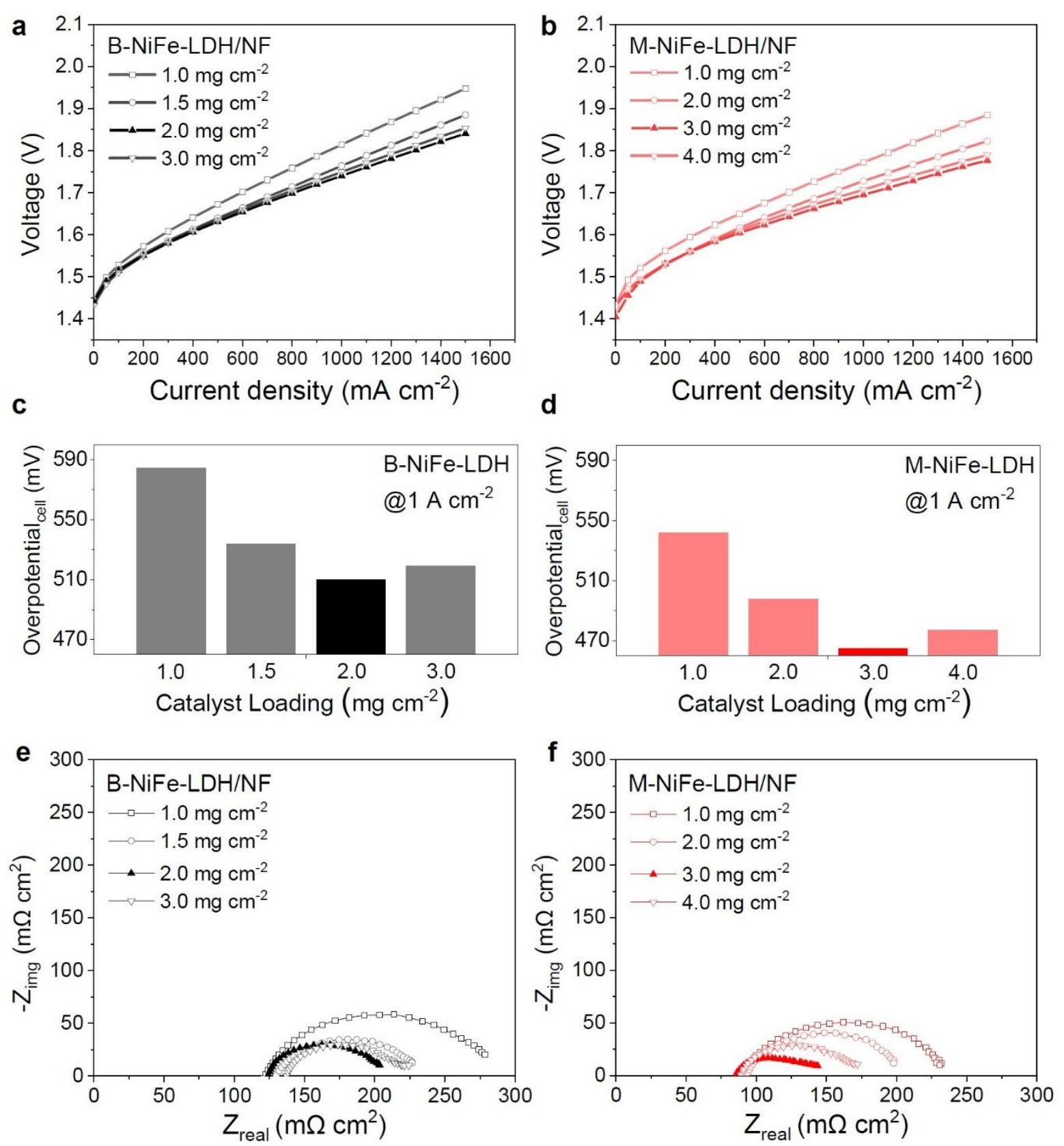

Figure S10. AEMWE results measured at $50{ }^{\circ} \mathrm{C}$ using B-NiFe-LDH/NF and M-NiFe-

LDH/NF prepared with various catalyst loadings. Polarization curves of (a) B-NiFe-LDH/NF and (b) M-NiFe-LDH/NF, the cell overpotential estimated at $1 \mathrm{~A} \mathrm{~cm}^{-2}$ for (c) B-NiFeLDH/NF and (d) M-NiFe-LDH/NF, and Nyquist plots from EIS measured at $1.7 \mathrm{~V}$ for (e) B$\mathrm{NiFe}-\mathrm{LDH} / \mathrm{NF}$ and (f) M-NiFe-LDH/NF as anodes. 

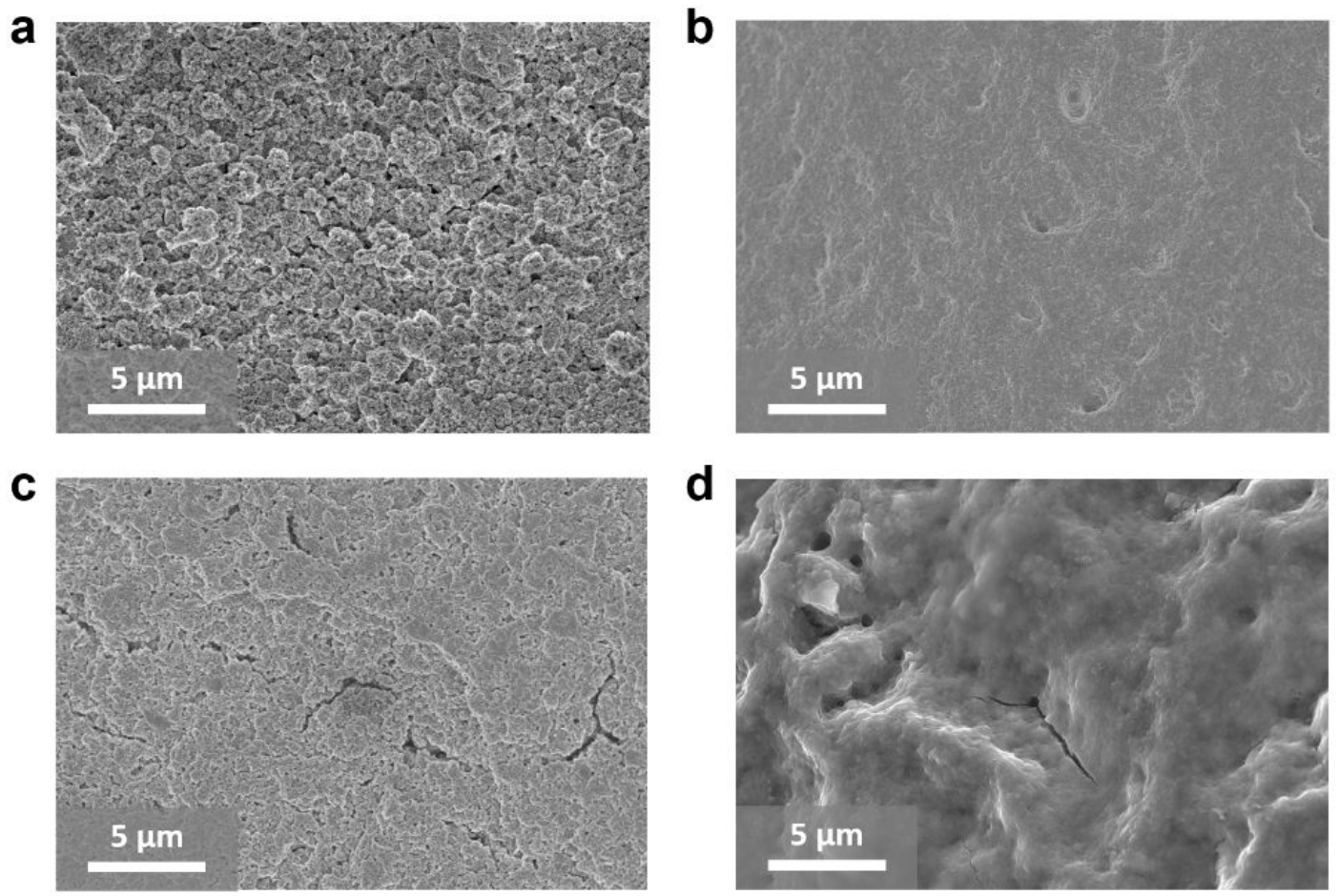

Figure S11. SEM images of (a) B-NiFe-LDH, and (b) M-NiFe-LDH spray-coated on Ni foam before the stability test. SEM images of (c) B-NiFe-LDH, and (d) M-NiFe-LDH spray-coated on Ni foam after the stability test. The stability test was carried out by galvanostatic operation at $1 \mathrm{~A} \mathrm{~cm}^{-2}$ at cell temperature of $50{ }^{\circ} \mathrm{C}$ for $50 \mathrm{~h}$. 

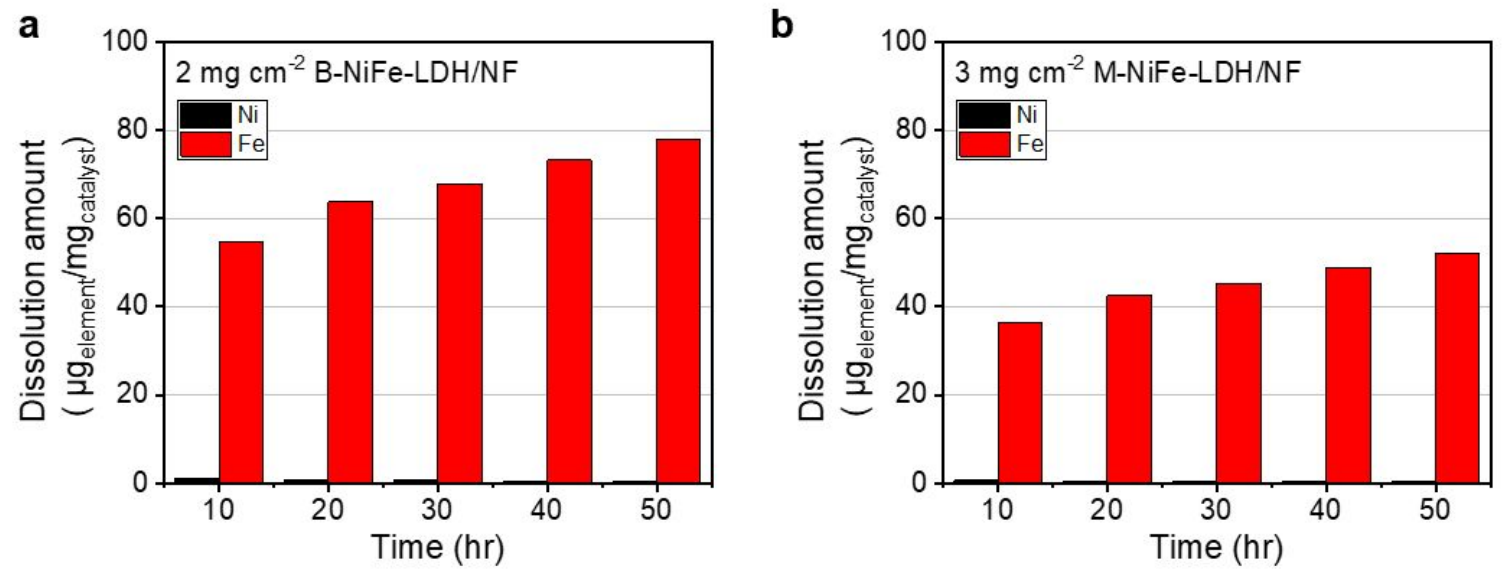

Figure S12. ICP-MS results for the amount of Ni \& Fe leached out from (a) B-NiFe-LDH/NF and (b) M-NiFe-LDH/NF electrodes during the stability test, normalized by the catalyst loading. The stability test was carried out by galvanostatic operation at $1 \mathrm{~A} \mathrm{~cm}^{-2}$ at cell temperature of $50{ }^{\circ} \mathrm{C}$ for $50 \mathrm{~h}$. 


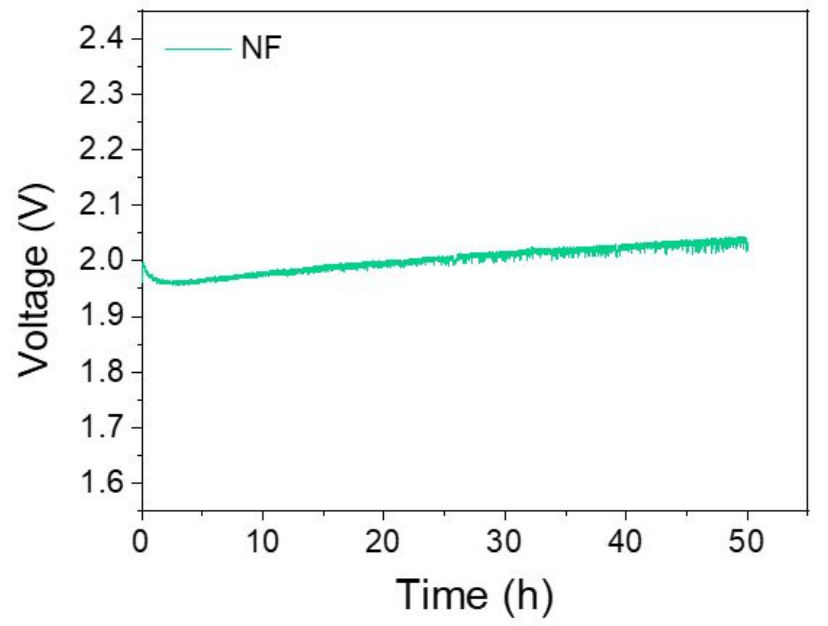

Figure S13. The result of AEMWE stability test for bare Ni foam measured at $1 \mathrm{~A} \mathrm{~cm}^{-2}$ and $50{ }^{\circ} \mathrm{C}$. 

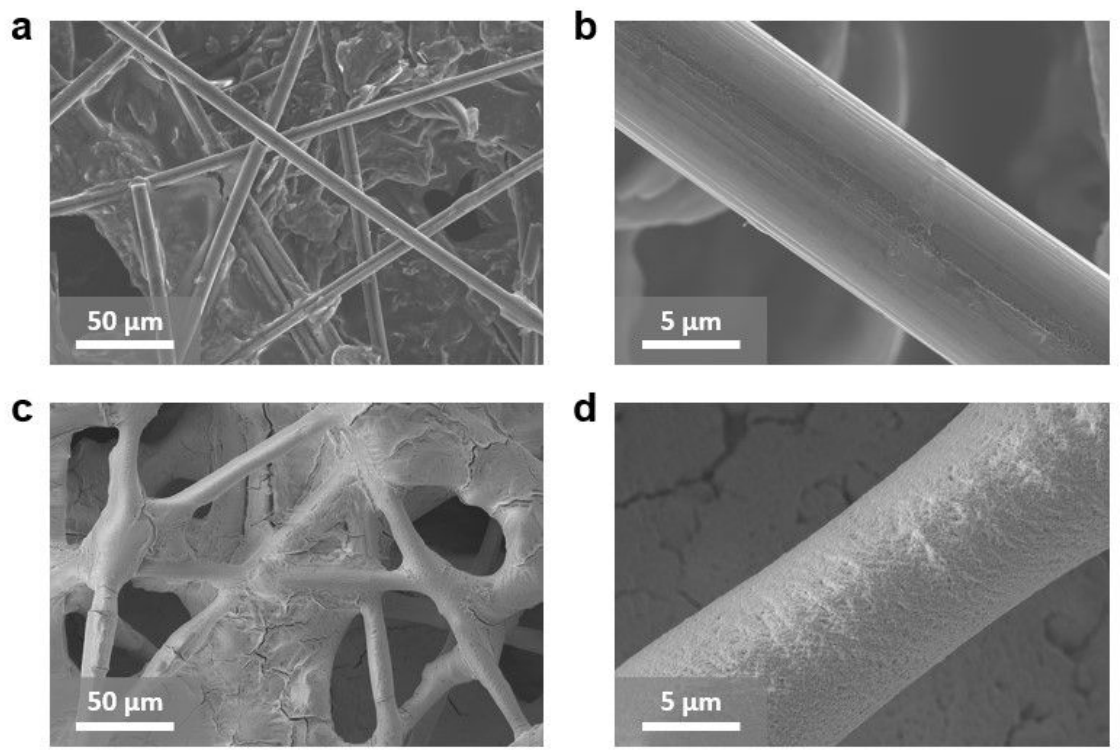

e
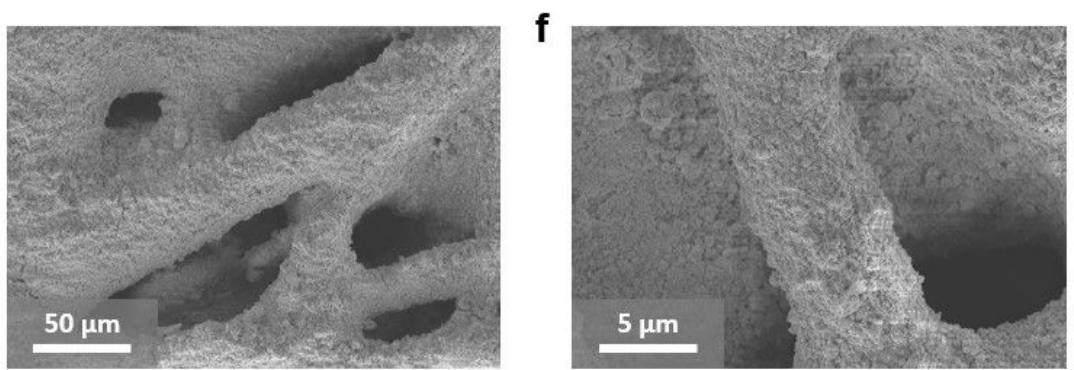

Figure S14. SEM images of (a), (b) bare carbon paper, (c), (d) M-NiFe-LDH sprayed on carbon paper, and (e), (f) B-NiFe-LDH sprayed on carbon paper. 


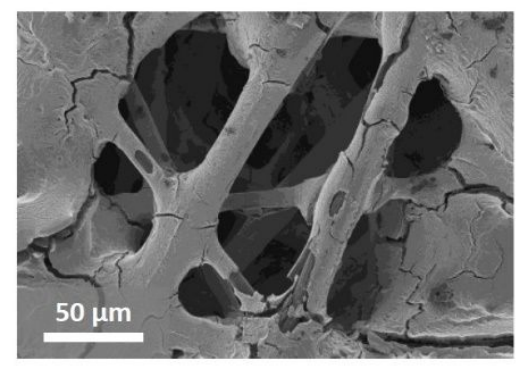

Figure S15. SEM images of M-NiFe-LDH sprayed on carbon paper after the stability test (galvanic operation at $1 \mathrm{~A} \mathrm{~cm}^{-2}$ for $50 \mathrm{~h}$ ) at $50{ }^{\circ} \mathrm{C}$. 

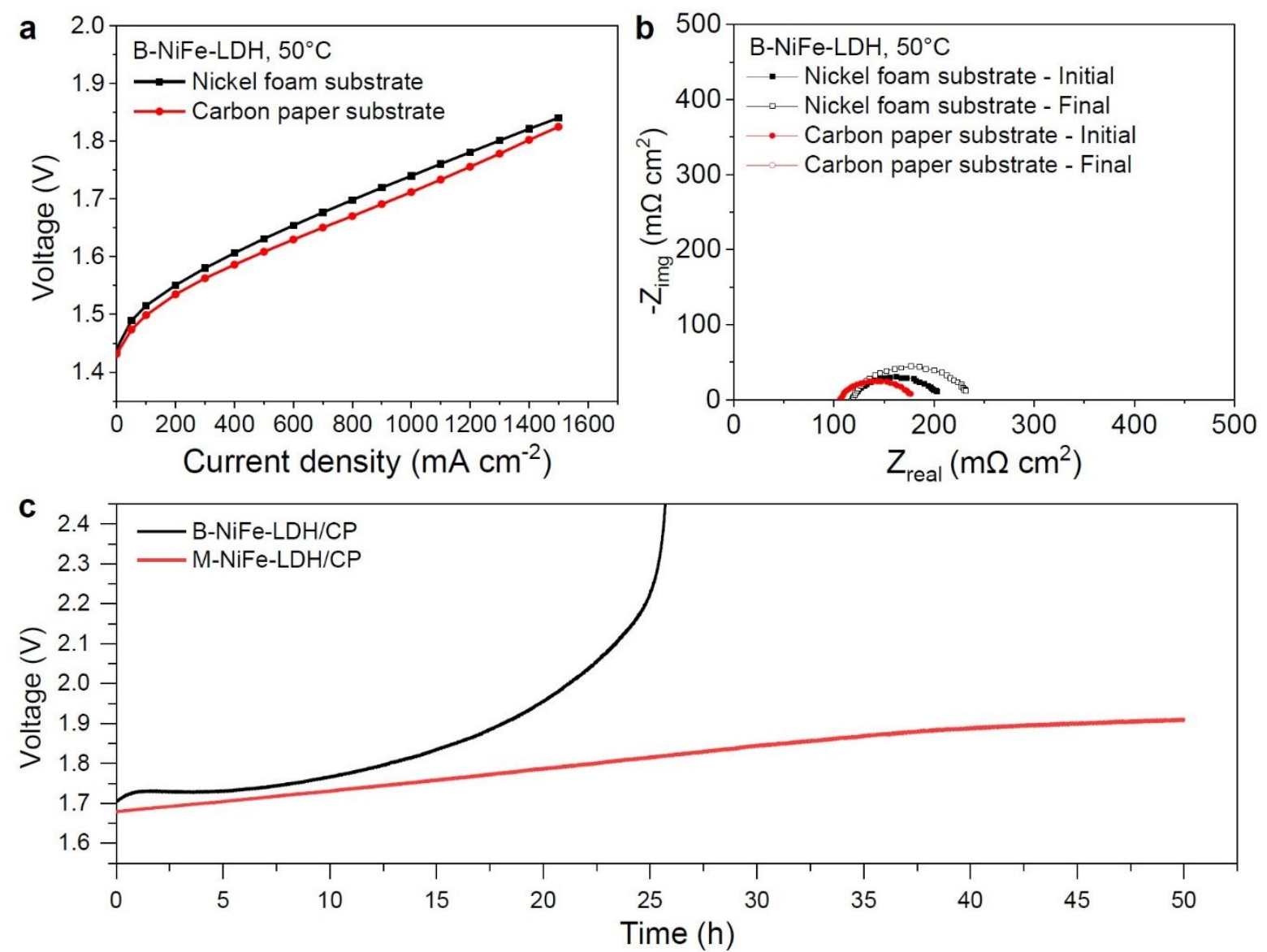

Figure S16. AEMWE (a) Polarization curves and (b) EIS before and after the stability test measured at $50{ }^{\circ} \mathrm{C}$ using B-NiFe-LDH $\left(2.0 \mathrm{mg} \mathrm{cm}^{-2}\right)$ on Ni foam or carbon paper. (c) The stability test results measured by galvanostatic operation at $1 \mathrm{~A} \mathrm{~cm}^{-2}$ at $50{ }^{\circ} \mathrm{C}$ for $\mathrm{B}-\mathrm{NiFe}-$ LDH on carbon paper and M-NiFe-LDH on carbon paper. 
Table S2. A summary of materials, conditions, and performances of state-of-art alkaline AEMWEs measured without carbonaceous materials at anode reported in recent 2 years (2019 \& 2020).

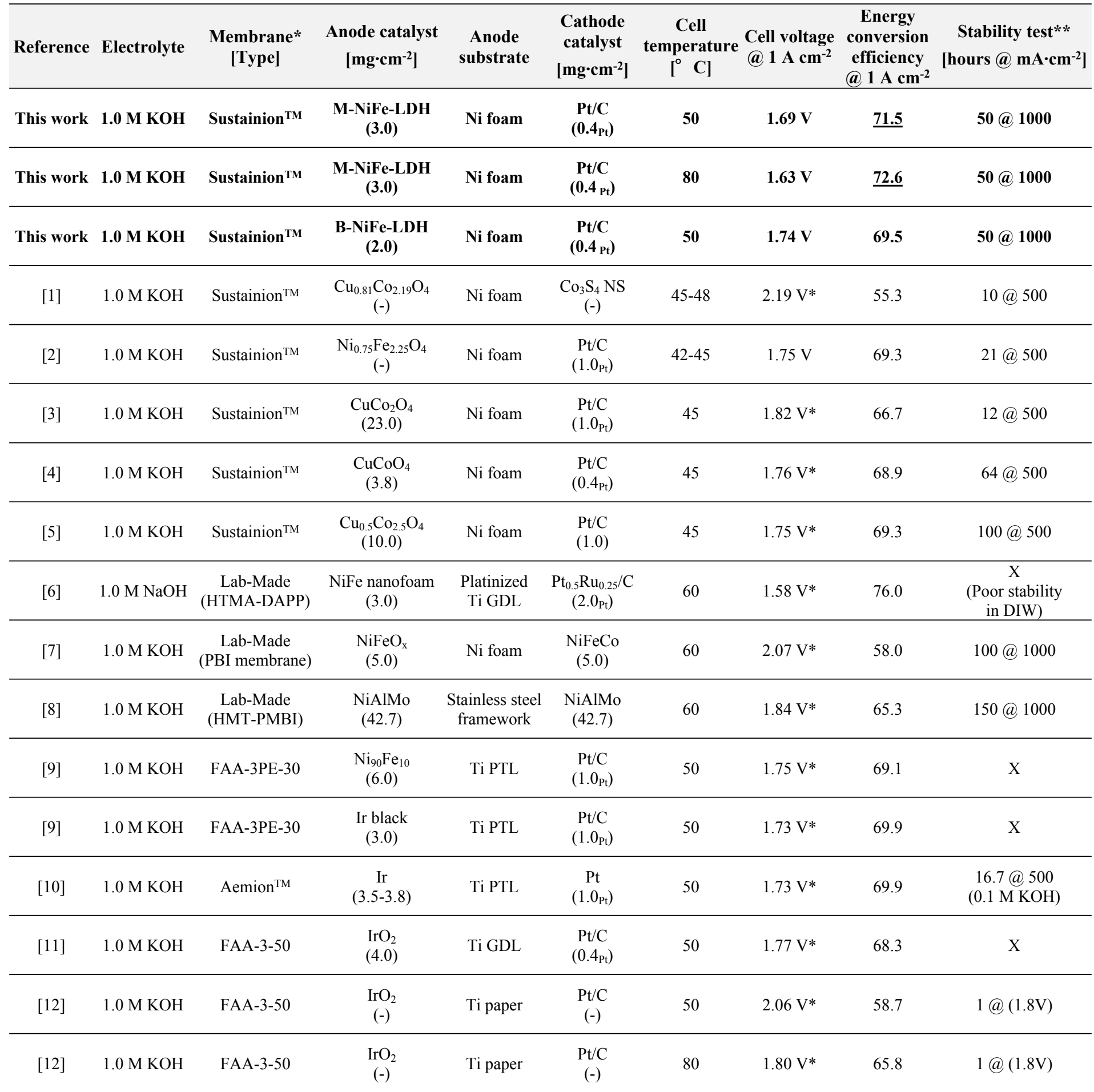

*Performance interpolated at current density of $1 \mathrm{~A} \mathrm{~cm}^{-2}$.

**Stability test at same conditions as cell performance measurement unless otherwise stated.

\section{References}


[1] Park, Y. S.; Lee, J. H.; Jang, M. J.; Jeong, J.; Park, S. M.; Choi, W.-S.; Kim, Y.; Yang, J.; Choi, S. M., $\mathrm{Co}_{3} \mathrm{~S}_{4}$ Nanosheets on Ni Foam via Electrodeposition with Sulfurization as Highly Active Electrocatalysts for Anion Exchange Membrane Electrolyzer. Int. J. Hydrog. Energy 2020, 45 (1), 36-45.

[2] Lee, J.; Jung, H.; Park, Y. S.; Woo, S.; Kwon, N.; Xing, Y.; Oh, S. H.; Choi, S. M.; Han, J. W.; Lim, B., Corrosion-Engineered Bimetallic Oxide Electrode as Anode for High-Efficiency Anion Exchange Membrane Water Electrolyzer. Chem. Eng. J. 2020, 127670.

[3] Park, Y. S.; Jang, M. J.; Jeong, J.; Park, S. M.; Wang, X.; Seo, M. H.; Choi, S. M.; Yang, J., Hierarchical Chestnut-Burr Like Structure of Copper Cobalt Oxide Electrocatalyst Directly Grown on Ni Foam for Anion Exchange Membrane Water Electrolysis. ACS Sustainable Chem. Eng. 2020, 8(6), 2344-2349.

[4] Park, Y. S.; Yang, J.; Lee, J.; Jang, M. J.; Jeong, J.; Choi, W.-S.; Kim, Y.; Yin, Y.; Seo, M. H.; Chen, Z.; Choi, S. M., Superior Performance of Anion Exchange Membrane Water Electrolyzer: Ensemble of Producing Oxygen Vacancies and Controlling Mass Transfer Resistance. Appl. Catal. B: Environ. 2020, 278, 119276.

[5] Jang, M. J.; Yang, J.; Lee, J.; Park, Y. S.; Jeong, J.; Park, S. M.; Jeong, J.-Y.; Yin, Y.; Seo, M.-H.; Choi, S. M.; Lee, K. H., Superior Performance and Stability of Anion Exchange Membrane Water Electrolysis: pH-Controlled Copper Cobalt Oxide Nanoparticles for the Oxygen Evolution Reaction. J. Mater. Chem. A 2020, 8(8), 42904299.

[6] Li, D.; Park, E. J.; Zhu, W.; Shi, Q.; Zhou, Y.; Tian, H.; Lin, Y.; Serov, A.; Zulevi, B.; Baca, E. D.; Fujimoto, C.; Chung, H. T.; Kim, Y. S., Highly Quaternized Polystyrene Ionomers for High Performance Anion Exchange Membrane Water Electrolysers. Nat. Energy 2020, 5(5), 378-385.

[7] Vincent, I.; Lee, E.-C.; Kim, H.-M., Highly Cost-Effective Platinum-Free Anion Exchange Membrane Electrolysis for Large Scale Energy Storage and Hydrogen Production. RSC Adv. 2020, 10 (61), 37429-37438.

[8] Wang, L.; Weissbach, T.; Reissner, R.; Ansar, A.; Gago, A. S.; Holdcroft, S.; Friedrich, K. A., High Performance Anion Exchange Membrane Electrolysis Using PlasmaSprayed, Non-Precious-Metal Electrodes. ACS Appl. Energy Mater. 2019, 2 (11), 7903-7912.

[9] Cossar, E.; Oyarce Barnett, A.; Seland, F.; Baranova, E. A., The Performance of Nickel and Nickel-Iron Catalysts Evaluated As Anodes in Anion Exchange Membrane Water 
Electrolysis. Catalysts 2019, 9(10), 814.

[10] Campagna Zignani, S.; Lo Faro, M.; Trocino, S.; Aricò, A. S., Investigation of NiFeBased Catalysts for Oxygen Evolution in Anion-Exchange Membrane Electrolysis. Energies 2020, $13(7), 1720$.

[11] Park, J. E.; Kang, S. Y.; Oh, S.-H.; Kim, J. K.; Lim, M. S.; Ahn, C.-Y.; Cho, Y.-H.; Sung, Y.-E., High-Performance Anion-Exchange Membrane Water Electrolysis. Electrochim. Acta 2019, 295, 99-106.

[12] Lim, A.; Kim, H.-J.; Henkensmeier, D.; Jong Yoo, S.; Young Kim, J.; Young Lee, S.; Sung, Y.-E.; Jang, J. H.; Park, H. S., A Study on Electrode Fabrication and Operation Variables Affecting the Performance of Anion Exchange Membrane Water Electrolysis. J. Ind. Eng. Chem. 2019, 76, 410-418. 
Note S1. Energy conversion efficiency calculation formula

The energy conversion efficiency can be calculated by following equations (S1) - (S3)

$$
\eta=\frac{E_{o}}{V}
$$

Where:

$$
\begin{gathered}
E_{o}=-\frac{\Delta G}{n F} \\
\Delta G=\Delta H-\mathrm{T} \Delta S
\end{gathered}
$$

$\eta: \quad$ Energy conversion efficiency

$\mathrm{E}_{0}: \quad$ Thermodynamically required voltage $\left(\mathrm{H}_{2} \mathrm{O} \rightarrow \mathrm{H}_{2}+1 / 2 \mathrm{O}_{2}\right)$

$\mathrm{V}$ : $\quad$ Measured voltage

$\Delta \mathrm{G}: \quad$ Gibbs free energy change

$\mathrm{n}$ : No. of electrons participating in above reaction $(\mathrm{n}=2)$

F: $\quad$ Faradaic constant $\left(96,485 \mathrm{C} \mathrm{mol}^{-1}\right)$

$\Delta \mathrm{H}: \quad$ Change in enthalpy during water electrolysis $\left(-285.8 \mathrm{~kJ} \mathrm{~K}^{-1} \mathrm{~mol}^{-1}\right)$

$\Delta \mathrm{S}: \quad$ Change in entropy during water electrolysis $\left(-163.34 \mathrm{~J} \mathrm{~K}^{-1} \mathrm{~mol}^{-1}\right)$ 
Table S3. A summary of materials, conditions, and performances of state-of-art alkaline AEMWEs measured with carbonaceous materials at anode reported in recent 2 years $(2019$ \& 2020).

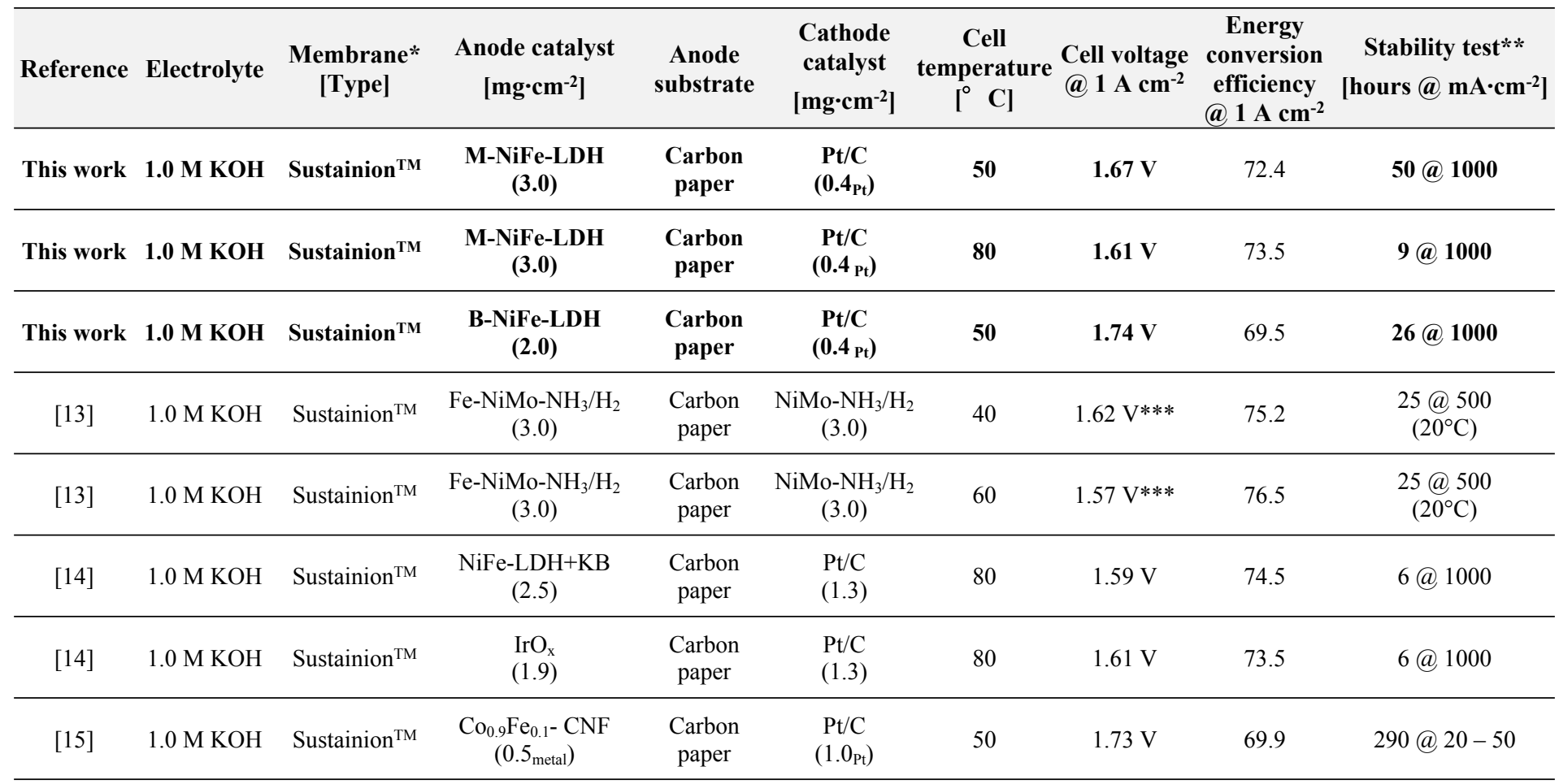

*Performance interpolated at current density of $1 \mathrm{~A} \mathrm{~cm}^{-2}$.

**Stability test at same conditions as cell performance measurement unless otherwise stated.

$* * *$ Performance extrapolated at current density of $1 \mathrm{~A} \mathrm{~cm}^{-2}$.

KB: Ketjen Black (Conductive carbon additive)

CNF: Carbon nanofiber

\section{References}

[13] Chen, P.; Hu, X., High-Efficiency Anion Exchange Membrane Water Electrolysis Employing Non-Noble Metal Catalysts. Adv. Energy Mater. 2020, 10 (39), 2002285.

[14] Koshikawa, H.; Murase, H.; Hayashi, T.; Nakajima, K.; Mashiko, H.; Shiraishi, S.; Tsuji, Y., Single Nanometer-Sized NiFe-Layered Double Hydroxides as Anode Catalyst in Anion Exchange Membrane Water Electrolysis Cell with Energy Conversion Efficiency of $74.7 \%$ at $1.0 \mathrm{~A} \mathrm{~cm}^{-2}$. ACS Catal. 2020, 10 (3), 18861893.Chen, P.; Hu, X., High-Efficiency Anion Exchange Membrane Water Electrolysis Employing Non-Noble Metal Catalysts. Adv. Energy Mater. 2020, 10 (39), 2002285.

[15] Kang, S.; Ham, K.; Lee, J., Moderate Oxophilic CoFe in Carbon Nanofiber for the Oxygen Evolution Reaction in Anion Exchange Membrane Water Electrolysis. Electrochim. Acta 2020, 353, 136521. 
